\title{
THE INFLUENCE OF THE PHOTOIONIZING RADIATION SPECTRUM ON METAL-LINE RATIOS IN LY $\alpha$ FOREST CLOUDS
}

\author{
Mark L. GirouX AND J. Michael Shull \\ Center for Astrophysics and Space Astronomy, Department of Astrophysical, Planetary, and Atmospheric Sciences. \\ University of Colorado, Campus Box 389 . Boulder, Colorado 80309 \\ Electronic mail: giroux@shapley.colorado.edu \\ Received 1996 Dectmber 20; revised 1997 Jamuary 17
}

\begin{abstract}
Recent measurements of Si IV/C IV ratios in the high-redshift Ly $\alpha$ forest [Songaila \& Cowie, AJ, 112. 335 (1996a); Savaglio et al., A\&A (in press) (1997)] have opened a new window on chemical enrichment and the first generations of stars. However, the derivation of accurate Si/C abundances requires reliable ionization corrections, which are strongly dependent on the spectral shape of the metagalactic ionizing background and on the "local effects" of hot stars in nearby galaxies. Recent models have assumed power-law quasar ionizing backgrounds plus a decrement at 4 Ryd to account for $\mathrm{He}$ II attenuation in intervening clouds. However, we show that realistic ionizing backgrounds based on cosmological radiative transfer models produce more complex ionizing spectra between 1-5 Ryd that are critical to interpreting ions of $\mathrm{Si}$ and $\mathrm{C}$. We also make a preliminary investigation of the effects of $\mathrm{He}$ II ionization front nonoverlap. Because the attenuation and reemission by intervening clouds enhance Si IV relative to $C$ IV. the observed high Si IV/C IV ratios do not require an unrealistic Si overproduction $\left[\mathrm{Si} / \mathrm{C} \geqslant 3(\mathrm{Si} / \mathrm{C})_{\mathrm{C}}\right]$. If the ionizing spectrum is dominated by "local effects" from massive stars, even larger Si IV/C IV ratios are possible. However, unless stellar radiation dominates quasars by more than a factor of 10 , we confirm the evidence for some $\mathrm{Si}$ overproduction by massive stars; values $\mathrm{Si} / \mathrm{C} \approx 2(\mathrm{Si} / \mathrm{C})_{\odot}$ fit the measurements better than solar abundances. Ultimately, an adequate interpretation of the ratios of C IV, Si IV, and C II may require hot, collisionally ionized gas in a multiphase medium. (1) 1997 American Astronomical Society: [S0004-6256(97)00205-7]
\end{abstract}

\section{INTRODUCTION}

The detection of metals associated with the "high" column density $\left[N(\mathrm{H} \mathrm{I})>10^{145} \mathrm{~cm}^{-2}\right]$ Ly $\alpha$ forest of absorbers in quasar spectra (Cowie et al. 1995; Tytler et al. 1995) provides new challenges to the understanding of the nature and origin of these absorbers. The contamination by metals must now be accounted for in models for cloud evolution. It is also possible to perform a detailed analysis of the thermodynamic and ionization state of these absorbers, which have previously been probed only by their hydrogen Ly $\alpha$ absorption and the integrated effect of their He II Ly $\alpha$ absorption (Giroux et al. 1995). If the clouds are photoionized, the detection of C II, C IV, and Si IV may constrain the shape of the metagalactic radiation spectrum and indicate the epoch of dramatic changes in the ionizing radiation field at $z>3$ (Songaila \& Cowie 1996a; Savaglio et al. 1997).

The multiple $\mathrm{C}$ IV lines associated with a single $\mathrm{H} \mathrm{I}$ absorption complex (Songaila \& Cowie 1996a) belies a simple picture of a homogeneous cloud. These absorbers, like Lyman limit systems, may better be interpreted with multiphase models (Giroux et al. 1994; Petitjean et al. 1996; Haehnelt et al. 1997; Hellsten et al. 1997) that may include hot, collisionally ionized gas. We defer such interpretation to a later

'Also at JILA, University of Colorado and National Institute of Standards and Technology. study, focusing here on the tradition of analyzing metal-line ratios with single-phase photoionization models. In this way, we can isolate the effects of the incident spectrum on species with ionization potentials in the range 1-5 Ryd (e.g.. C III. C IV, Si III, Si IV). For example, Songaila \& Cowie (1996a) argue that the observed increase in the Si IV/C IV ratio at $z>3.1$ suggests that the He III regions around ionizing sources may not have overlapped by that epoch, cutting off all radiation with $h v>54.4 \mathrm{eV}$. Here. we primarily consider models for the averaged metagalactic background in which He II ionization fronts have overlapped before $: \approx 4$. We do consider a limiting case in which no photons above 4 Ryd are present in the background, as well as a calse where we restore the much less heavily attenuated $x$-ray hackground.

In the past decade, metal-line ratios in Lyman Limit $\left(N_{\mathrm{H}} \geq 10^{17} \mathrm{~cm}^{-2}\right)$ absorbers have been used to prohe the shape of the ionizing spectrum, and to estimate the intrinsic metallicity of the absorbers (cf. Bergeron \& Stasinska 1986 : Sargent et al. 1988; Steidel et al. 1988; Steidel \& Sirgent 1989; Bergeron \& Ikeuchi 1990; Miralda-Escudé \& Ostriker 1990); Donahue \& Shull 1991; Madau 1992). With the aldvent of new spectrographs on large telescopes, the lower $N_{11}$ ahsorbers can be exploited in the same way. These absorbers hold a special interest, because the source of their metal enrichment is still unclear. While the metal lines in Lyman limit absorbers have been associated with the haloes of bright galaxies, the number of Lya forest absorbers greatly 
exceeds the corresponding number of bright galaxies, and the Ly $\alpha$ absorbers do not seem to show the same clustering properties as galaxies.

As yet, metal lines have only been associated with "highcolumn" absorbers $\left[N(\mathrm{H} \mathrm{I})>10^{1.5 .5} \mathrm{~cm}^{-2}\right]$, which numerically are a small fraction of the $\mathrm{Ly} \alpha$ forest. It is still possible that these clouds may represent an overlap of the highcolumn end of the pristine Ly $\alpha$ forest clouds and the lowcolumn end of the metal enriched clouds associated with galactic haloes. As a result some, if not all, of the metal-line absorbers may still be associated with the galaxies that contain the stars responsible for their metals. For example, over a Hubble time at $z=3$, galactic outflows at $300 V_{300} \mathrm{~km} \mathrm{~s}^{-1}$ will transport heavy elements a distance

$$
d \leqslant(250 \mathrm{kpc}) V_{3(0)} h^{-1}\left(\frac{1+z}{4}\right)^{-3 / 2}
$$

for a Friedmann universe with $H_{0}=\left(100 \mathrm{~km} \mathrm{~s}^{-1}\right) h$ and $\Omega_{0}$ $=1$. In practice, the period of heavy element injection may have lasted only $10^{8} y r$, and the metals could move distances of only $30-50 \mathrm{kpc}$ from their sources (bright galaxies, dwarf galaxies, globular clusters).

As Madau \& Shull (1996) have emphasized, metal enrichment of the Ly $\alpha$ absorbers implies a substantial Lyman continuum ( $\mathrm{LyC}$ ) emission accompanying the star formation at $z \geq 3.5$. Thus, a large fraction of the metagalactic background at high redshift could be due to massive stars, a point also made by Giroux \& Shapiro (1996) and Savaglio et al. (1997). Furthermore, the absorbers with metal lines may be sufficiently close to the local sources that the incident radiation field is dominated by local hot-star radiation. In contrast, if the metal enrichment of the Ly $\alpha$ forest arises from Population III stars at $z>10$ (Couchman 1985; Ostriker \& Gnedin 1996), the stellar ionizing radiation will be greatly attenuated by intergalactic absorption, and the metagalactic background at $z=3$ will be dominated by the harder spectrum of quasars. Because metal-line systems would then be less likely to be associated with nearby sources of stellar radiation, their absorption-line ratios would be more representative of the metagalactic ionizing background.

Preliminary analyses (Songaila \& Cowie 1996a; Savaglio et al. 1997; Haehnelt 1997) of the Ly $\alpha$ forest clouds have assumed photoionization by simple power-law ionizing spectra. The only way these models account for filtering of the ionizing source spectrum by intervening clouds is to include a decrement at $\nu_{\mathrm{He}}$ (4 Ryd). More realistic models include several additional effects. First, when absorption due to intervening clouds is included, the background spectrum just above the $\mathrm{HI}$ and $\mathrm{He} I \mathrm{I}$ ionization edges shows a flatter power law than that of the sources (Miralda-Escude \& Ostriker 1990): Madau \& Meiksin 1994; Giroux et al. 1995). Second. Haardt \& Madau (1996) and Fardal et al. (1997) show, using cosmological radiative transfer, that cloud emission by recombination to the $\mathrm{HI}$ and $\mathrm{He} I \mathrm{I}$ ground states and He II Lycr and two-photon radiation alters the extent of the decrement at $\nu_{\mathrm{Hc} \text { II }}$ and changes the shape of the spectrum between 1 and 5 Ryd. Including a more realistic spectral shape will alter the relative populations of metal ions with edges in the 1-5 Ryd range even if the ionizing sources are all assumed to be quasars with intrinsic power-law ionizing spectra.

In this paper, we consider the extent to which these alterations of the assumed shape of the incident radiation field affect the interpretation of the metallicity of these absorbers and the nature of the photoionizing sources. We use the data sets of Songaila \& Cowie (1996a) and Savaglio et al. (1997), which include Si IV and CIV lines associated with higher column density Ly $\alpha$ forest clouds. Songaila \& Cowie (1996a) provide an important subsample for which C II lines are also observed.

\section{THE METAGALACTIC RADIATION BACKGROUND}

\subsection{Ionizing Sources}

Assumptions about the nature of the ionizing sources of the metagalactic background are uncertain, since the intrinsic EUV spectra of quasars are not well defined. For simplicity, we assume that all quasars have constant power-law spectra $F_{y} \propto \nu^{-\alpha_{s}}$. Our choice of spectral index, $\alpha_{s}=1.8$, is consistent with $H S T$ observations of the EUV spectrum of quasars (Zheng et al. 1997) for radio-quiet quasars at $z=1.5-2.0$. As discussed by Fardal et al. (1997), if the ionizing background (1-5 Ryd) is dominated by these quasars, the He II Ly $\alpha$ absorption optical depth $\tau_{\mathrm{He} \mathrm{II}} \approx 1$ at $z=3$. For clarity, we denote the cloud-filtered, quasar-dominated mean intensity by $J_{Q, f}(\nu)$.

The characteristic spectrum of stellar sources in starburst galaxies is also not well known. Most models agree (e.g., Bruzual \& Charlot 1993) that few photons are present with frequency above $54.4 \mathrm{eV}$, and that the spectrum falls off above $h \nu \approx 45 \mathrm{eV}$. Recent line-blanketed, non-LTE hot-star models (Gabler et al. 1992; Sellmaier et al. 1996; Schaerer $\&$ de Koter 1996) vary considerably in the amount of radiation in the He I continuum $(24.6<h \nu<54.4 \mathrm{eV})$ they predict, compared to the LTE line-blanketed models of Kurucz (1992). An ongoing uncertainty, for our purposes, is the lack of low-metallicity stellar models, which are more appropriate for high-redshift starburst galaxies. For the present work, we use a starburst spectrum (Sutherland \& Shull 1997) discussed in the context of the ionizing spectrum of metalforming galaxies by Madau \& Shull (1996) (see their Fig. 3). In brief, it is a time integrated spectrum of a Gaussian starburst of width $2 \sigma=4 \mathrm{Myr}$ containing $5000 . \mathrm{H}_{\odot}$ of stars between 8 and 85 . $\mathscr{H}_{\odot}$ with IMF slope $\Gamma=1.6$, where $d N$ $(>. \mathscr{H}) / d . \mathbb{H} \times \cdot \mathbb{H}^{-\mathrm{I}}$ and $N(>, \mathscr{H})$ is the number of stars with mass greater than. $/$.

Estimates of the mean metagalactic ionizing intensity at $z>2$, based on the statistical diminution in the number of Ly $\alpha$ forest lines close to quasars (the proximity effect), indicate a specific intensity at the hydrogen Lyman limit, $\nu_{\mathrm{H}}$, of $J_{-21}=0.7-1.5$, where $J_{-21}$ is in units of $10^{-21} \mathrm{ergs} \mathrm{cm}^{-2} \mathrm{~s}^{-1} \mathrm{~Hz}^{-1} \mathrm{sr}^{-1}$ (Cooke et al. 1997). If all Ly $\alpha$ absorbers possess a metallicity of $Z=0.01 Z_{\odot}$ by redshift $z \approx 3.5$, the stellar ionizing photons associated with the production of the metals may be comparable to the ionizing photons produced by quasars (Madau \& Shull 1996; Giroux \& Shapiro 1996). If these ionizing photons are produced at higher redshift (e.g., Population III stars at $z>5$ ) they will be 
highly attenuated by IGM absorption at $z>4$. A further uncertainty is the fraction, $\left\langle f_{\text {exc }}\right\rangle$, of ionizing photons that successfully escape from the galaxy in which the stars reside (Dove \& Shull 1994). It does seem possible, though, that stellar sources could be an important contributor to the metagalactic background, which could dominate in regions local to starburst galaxies.

For example, the total Lyman continuum production rate of the Milky Way was recently inferred from COBE observations of $[\mathrm{N} \mathrm{II}] 205 \mu \mathrm{m}$ emission to be $3.5 \times 10^{53}$ photons $\mathrm{s}^{-1}$ (Bennett et al. 1994), and most starburst galaxies exceed the value $S=\left(10^{54}\right) S_{54}$ photons $s^{-1}$. Assuming that a substantial fraction, $\left\langle f_{\text {esc }}\right\rangle \geqslant 0.1$, of these photons escape these starburst galaxies, the mean photoionization rate of hydrogen due to the galactic source may exceed $J_{-21}$ $=1$ if the absorber lies within a distance

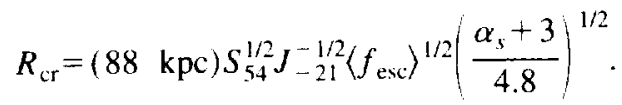

Here, we have assumed the QSO spectra in the range 1-5 Ryd to be power laws with spectral index $\alpha_{s} \approx 1.8$.

In this paper, we account for a stellar contribution to the mean intensity falling upon our absorbers in a global way. We assume that up to $2 / 3$ of the ionizing emissivity from primary sources is stellar, as opposed to secondary sources due to recombination radiation from clouds. The remainder of the primary emissivity is then from AGN. We then compute the radiative transfer of primary and secondary sources through the IGM to derive the metagalactic ionizing background, $J_{S Q, f}(\nu)$. The mean intensities $J_{Q, f}$ (pure QSO sources) and $J_{S Q, f}$ (stars + QSOs) therefore bracket our estimates of the contribution of stellar sources to the uniform metagalactic background, $J_{M B}(v)$. However, even if the metagalactic ionizing background is quasar dominated, the absorber may primarily see a local unfiltered source of stellar photons, $J_{L}(\nu)$. We consider models which assume $J_{L} / J_{\mathrm{MB}}=5,10,20$ at $\nu=\nu_{\mathrm{H}}$ for $J_{\mathrm{MB}}=J_{Q, f}$ and $J_{L} / J_{\mathrm{MB}}=3$, $5,10,20$ for $J_{\mathrm{MB}}=J_{S Q, f}$.

\subsection{He II loniaation Fronts}

Within a picture of ionizing sources turning on in a neutral medium, Miralda-Escudé \& Rees (1994) made the important suggestion that the He II I-front which propagates away from sources with a sufficiently soft ionizing spectrum can lag the hydrogen I-front. Madau \& Meiksin (1994) showed that, if $\alpha_{x}=1.9$, the overlap of He II I-fronts could be delayed to as late as $z=3$. As a result, both Jakobsen $e t$ al. (1994) and Songaila \& Cowie (1996a) suggested that, at $z=3.1$, He II I-fronts around the dominant ionizing sources may not have overlapped. Although a full treatment of overlapping He II I-fronts is beyond the scope of this paper, we can make a simple estimate of the ratio of I-front velocities for $\mathrm{H} \mathrm{I}$ and $\mathrm{He}$ II. From the flux-limiting equations governing I-front propagation (Shapiro \& Giroux 1987; Donahue \& Shull 1987), the velocity relative to the local Hubble flow is given by

$$
\left(\frac{d r_{i}}{d t}-H r_{i}\right)=\frac{S_{i}}{4 \pi r_{i}^{2} n_{i}}
$$

where $i$ refers either to the $\mathrm{H} l$ ionizing front ( 1 Ryd continuum) or to the He 11 ionizing front (4 Ryd continuum). (The $\mathrm{He} \mathrm{l}$ ionizing front is assumed to be coincident with the $\mathrm{H} \mathrm{I}$ front, and we neglect recombinations and attenuation within the ionized zone.) Here, $S_{i}$ (photons $\mathrm{s}^{-1}$ ) represents the photon production rates of the source in the $\mathrm{H} \mathrm{I}$ and $\mathrm{He} I \mathrm{I}$ continua, $n_{i}$ is the density of $\mathrm{H} \mathrm{I}$ or $\mathrm{He} \mathrm{II}$, and $r_{i}$ is the front distance measured from the source.

The propagation speeds of the $\mathrm{H} \mathrm{I}$ and $\mathrm{He}$ II ionization fronts will be governed by the "flux-to-density" ratio. $\left(S_{i} / n_{i} r_{i}^{2}\right)$, at 1 and 4 Ryd. For a QSO spectrum with powerlaw index $\alpha_{s}$, one finds that $S_{\mathrm{He} \mathrm{II}} / S_{\mathrm{Hl}}=4^{-\alpha_{s}}$. The primordial helium abundance by mass has been estimated at values $Y_{P}=0.231 \pm 0.006$ (Skillman \& Kennicutt 1993) and $Y_{P}=0.232 \pm 0.003$ (Olive \& Steigman 1995), while Copi et al. (1994) have suggested a $2 \sigma$ concordance range of $Y_{P}$ $=0.221-0.243$ from theoretical models of Big-Bang nucleosynthesis. If helium has a cosmological abundance. $n_{\mathrm{He}} / n_{\mathrm{H}}=0.0785$ by number ( $Y=0.239$ by mass), the flux-todensity ratios at a fixed radius $r_{i}$ are equal for

$$
\alpha_{s}=-\frac{\ln \left(n_{\mathrm{He}} / n_{\mathrm{H}}\right)}{\ln 4} \approx 1.84
$$

At this critical spectral index, the $\mathrm{H}$ I and He II ionization fronts will coincide. Interestingly, this critical index is very close to the mean index found by Zheng et al. (1997) for radio-quiet quasars. For AGN with harder spectra ( $\alpha_{3}$ $<1.84$ ), the $\mathrm{He}$ II ionization front will precede the $\mathrm{H} I$ front by a small amount, equivalent to a few optical depths in the 4 Ryd continuum. However, for those AGN with softer spec$\operatorname{tra}\left(\alpha_{3}>1.84\right)$, the He II front will propagate at a lower speed and lie well within the $\mathrm{H}$ I front. For example, Zheng et al. (1997) found that radio-loud quasars had a mean index $\alpha_{s} \approx 2.2$. In this case, the regions outside the He II fronts will see no 4 Ryd continuum radiation.

We therefore consider a limiting case in which all photons above 4 Ryd are removed from the metagalactic background spectrum. We also consider an intermediate case, arising from the fact that quasars emit radiation at least into the $x$-ray range of energies, so that high-energy photons will not be strongly attenuated, even in regions where helium is entirely in the He II ionization stage. In the Ly $\alpha$ clouds. we estimate the effect of the presence of these high-energy photons by presuming that $H$ II regions have overlapped by : $=4$, and that the Ly $\alpha$ forest clouds. whose distribution in $N_{\mathrm{H}}$ is known (see Sec. 2.2), have a maximal $\mathrm{He} \mathrm{I} / \mathrm{H}$ I ratio. In interpreting He II Gunn-Peterson measurements, several groups (Madau \& Meiksin 1994; Giroux et al. 1995) tind that the ratio, $\eta=N(\mathrm{He}[\mathrm{I}) / N(\mathrm{H} \mathrm{J})$, must he $50-100)$ in order to explain the $\lambda<304 \AA$ absorption as He II line opacity in the $\mathrm{H}$ । Ly $\alpha$ forest. This large ratio arises because He II is more difficult to photoionize than $\mathrm{H} \mathrm{I}$.

The $H$ neutral fraction $f_{H_{1}}$ is always less than $\left(n_{\mathrm{He}} / n_{\mathrm{H}}\right) \eta^{-1}$, where $\eta=N(\mathrm{He}$ II $) / N(\mathrm{H} \mathrm{I})$ within an absorbing cloud. Thus, the maximal ratio $\eta_{\text {max }}$ is bounded by limits on the baryon density from Big Bang nucleosynthesis (BBN), $\Omega_{b} h^{2} \leqslant 0.024$ (Copi et al. 1994). At $z=3.4$, if only absorbers with $N_{\mathrm{H} !}<10^{15} \mathrm{~cm}^{-2}$ are considered to contribute to $\Omega_{H_{1}}$. we find $\eta_{\text {mix }}<50(0)$. This limit produces a local 
TABLE I. Significant multistage metals

\begin{tabular}{ll}
\hline \hline Species & \multicolumn{1}{c}{$E_{\text {th }}($ Ryd $)$} \\
\hline C II,II,IV & $24.38,47.89,64.49$ \\
N I,II,II,IV & $14.53,29.60,47.45,77.47$ \\
O I,H,III & $13.62,35.12,54.94$ \\
Ne I,II.III & $21.57,40.96,63.46$ \\
Si II,III,IV,V & $16.35,33.49,45.14,166.77$ \\
Al II,III & $18.83,28.45$ \\
S II,II,IV,V & $23.33,34.83,47.3,72.68$ \\
Fe II,III,IV & $16.19,30.65,54.8$ \\
\hline \hline
\end{tabular}

continuum optical depth at 4 Ryd, $d \tau / d z>180$ at $z=3.4$, falling to 4 by $\nu=25 \nu_{\mathrm{H}}$, the same continuum optical depth as that at 1 Ryd. As a result, for this model with nonoverlapping He II I-fronts, we only remove photons between 4 and $25 \mathrm{Ryd}$, retaining metagalactic radiation background for $\nu>25 \nu_{\mathrm{H}}$. This is a more conservative estimate than if all $\mathrm{He}$ is assumed to be in He II and distributed uniformly.

\subsection{Cloud Opacity/Emission}

For a random spatial distribution, the local continuum optical depth of the intervening absorbers is given by (Paresce et al. 1980)

$$
\frac{d \tau(\nu)}{d z}=\int_{0}^{\infty} \frac{\partial^{2} N_{c}}{\partial N_{\mathrm{H},} \partial z}\left[1-e^{-N_{\mathrm{H}} \sigma_{\mathrm{efl}}(\nu)}\right] d N_{\mathrm{H} \mathrm{l}},
$$

where, for clouds composed of $\mathrm{H}$ and $\mathrm{He}$,

$$
\sigma_{\mathrm{eff}}(\nu)=\sigma_{\mathrm{H} I}(\nu)+\left(\frac{N_{\mathrm{HeI}}}{N_{\mathrm{HI}_{\mathrm{I}}}}\right) \sigma_{\mathrm{He} \mathrm{I}}(\nu)+\left(\frac{N_{\mathrm{He} \mathrm{II}}}{N_{\mathrm{HI}}}\right) \sigma_{\mathrm{He} \mathrm{II}}(\nu),
$$

and $\partial^{2} N_{c} / \partial N_{\mathrm{H}} \partial z$ is the distribution of absorbers in column density and redshift. This distribution has often been parameterized by a function of the form

$$
\frac{\partial^{2} N_{c}}{\partial N_{\mathrm{H}} \partial z}=A(1+z)^{\gamma} N_{\mathrm{H}_{\mathrm{I}}}^{-\beta} \text {. }
$$

for $N_{l}(\mathrm{H} \mathrm{l}) \leqslant N_{\mathrm{H} I}\left(\mathrm{~cm}^{-2}\right) \leqslant N_{u}(\mathrm{H} \mathrm{I})$. Using new line lists from Keck data, Fardal et al. (1997) have completed a review of the statistics available on Ly $\alpha$ absorbers, Lyman Limit Systems, and damped Ly $\alpha$ systems. They suggest that the distribution of absorbers is best represented by a broken power law in $N_{\mathrm{HI}}$. If

$$
\frac{\partial^{2} N_{c}}{\partial N_{17} \partial z}=A_{i}(1+z)^{\gamma} N_{17}^{-\beta_{i}}
$$

where $N_{17}=N_{\mathrm{H}_{\mathrm{I}}} /\left(10^{17} \mathrm{~cm}^{-2}\right), \gamma=2.585$, and $\beta_{i}$ and $A_{i}$ represent coefficients appropriate for a given range in $N_{17}$ (see Table 2). We adopted a preliminary model for this paper, but use of any of their final models does not affect our conclusions.

Just as absorption due to intervening clouds will strongly alter the background radiation spectrum, the reemission of ionizing radiation from the clouds will also affect the radiation (Haardt \& Madau 1996; Fardal et al. 1997). Recombinations in the clouds produce continuum radiation $(\mathrm{H}$ I Lyc, He II Lyc, He II Bac, and He II 2-photon) as well as He II
TABle 2. Absorber distribution.

\begin{tabular}{clc}
\hline \hline Range in $N_{17}$ & \multicolumn{1}{c}{$A_{i}$} & $\beta_{i}$ \\
\hline $10^{-5}-10^{-3}$ & 0.132 & 1.41 \\
$10^{-3}-0.02$ & $6.31 \times 10^{-3}$ & 1.85 \\
$0.02-10^{5}$ & $2.9 \times 10^{-2}$ & 1.46 \\
\hline
\end{tabular}

Ly $\alpha$ ( $\lambda 304$ ) line radiation. Accounting properly for the Lyc and $\mathrm{Bac}$ radiation is critical to the determination of the level of the mean intensity above 4 Ryd. While the line and 2-photon radiation are not important for that, they strongly alter the spectrum between 1 and 3 Ryd and affect the populations of ions with thresholds in that range (see Table 1). A complete discussion of the treatment of reemitted radiation used in computing the filtered spectra is given in Fardal $e t$ al. (1997).

\section{Ly $\alpha$ FOREST CLOUD MODELS}

As stated previously, we neglect the possibility that the absorbers consist of more than one thermal and ionization phase to concentrate on the effects of the shape of the photoionizing radiation spectrum. We model the Ly $\alpha$ forest absorbers using the photoionization code CLOUDY version 90 (Ferland 1996). The absorber clouds are modelled as planeparallel slabs of constant density, illuminated on both sides by the ionizing radiation field. We adopt a fiducial column density $N(\mathrm{H} \mathrm{l})=10^{15} \mathrm{~cm}^{-2}$ and assume a metallicity $Z$ $=10^{-2} Z_{\odot}$. We adopt the spectral shapes for the incident radiation discussed in Sec. 2.1 and vary the strength of the incident radiation through the ionization parameter, $U$ $=n_{\gamma} / n_{\mathrm{H}}$, where $n_{\gamma}$ and $n_{\mathrm{H}}$ are the number densities of ionizing photons and hydrogen respectively. Each curve in Figs. 2,4 , and 6 represents a set of photoionization models paired with the corresponding spectrum shown in Figs. 1, 3, and 5, respectively.

Although we focus this paper on the effect of the spectral shape, we have also explored the effect of varying the parameters assumed above. For the spectral shapes considered here, there is little change in the ratios $(\mathrm{C} I V / \mathrm{C} \mathrm{IV}$ is reduced by $\sim 10 \%$ and $\mathrm{Si} \mathrm{IV} / \mathrm{C} \mathrm{IV}$ is raised $\sim 10 \%)$ if $N(\mathrm{H} \mathrm{I})$ is increased by a factor of 10 , as long as the temperatures for the clouds are assumed to be consistent with the thermal equilibrium solution of the CLOUDY model. An increase (or decrease) by a factor of 3 in the assumed metallicity has a slightly larger effect. The C II/C IV fraction is reduced $20 \%-$ $30 \%$ with a factor of 3 reduction in metallicity and raised $8 \%-15 \%$ by a factor of 3 increase in metallicity. The ratio $\mathrm{Si}$ IV/C IV is raised as much as $10 \%$ and reduced by $6 \%$ or less with the same changes in metallicity. Constraints on the $\mathrm{H}$ l columns of the clouds preclude large reductions in the assumed density for one-phase photoionization models, to avoid excessive length scales for the clouds and to ensure that contributions to the baryon density from these $N\left(\mathrm{H}_{\mathrm{l}}\right)$ $\geq 10^{15} \mathrm{~cm}^{-2}$ absorbers do not exceed BBN limits.

We normally allow the temperature to be that solved for by CLOUDY, assuming thermal equilibrium between photoelectric heating and radiative cooling. If the clouds are actually cooler, for example, if they have expended some energy 


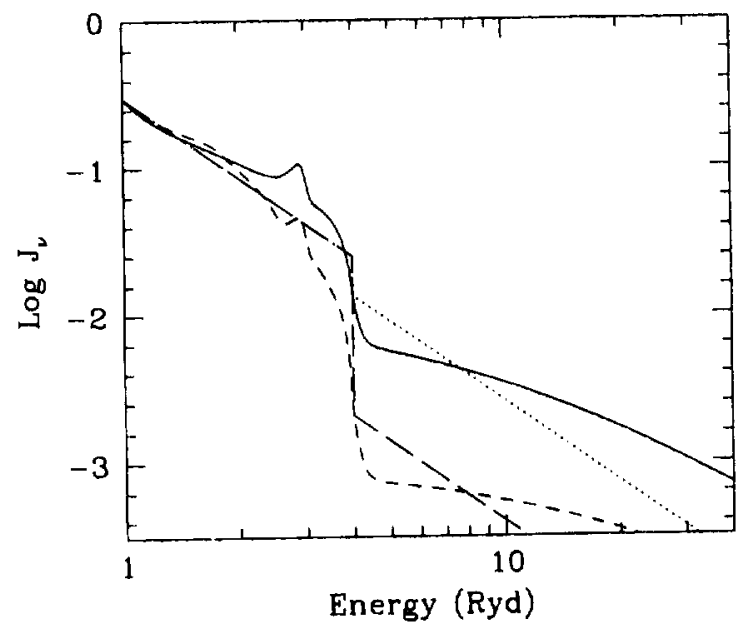

Fici. 1. Mean intensity, in relative units, vs energy in Ryd. All curves have been normalized to the same values at $v=\nu_{H}$ (1 Ryd). Solid curve assumes IGM-filtered sources with AGN $\left(\nu^{-1.8}\right)$ spectrum at $z=3.4$. Dotted curve assumes a mean intensity with $v^{-1.8}$ ahove $\nu=\nu_{\mathrm{H}}\left(1\right.$ Ryd) and $\nu=\nu_{\mathrm{He}}$ (4 Ryd), with decrease by a factor of 2 above $\nu_{\mathrm{ll} \mid \mathrm{H}}$, designed to give same ratio, $J(4 \mathrm{Ryd} / J$ (I Ryd), as the properly filtered case. Short-dashed curve assumes an IGM-filtered spectrum: 2/3 starburst (from Surtherland \& Shull 1997 ) and $1 / 3$ AGN. Long-dashed curve assumes $\nu^{-1.8}$ above $\nu=\nu_{11}$ and $\nu=\nu_{\mathrm{H} \cdot \| l}$. and a decreatse by a factor of 12 above $\nu_{\mathrm{He} \| 1 /}$. 0 give same ratio, $J(4 \mathrm{Ryd}) / J(\mid \mathrm{Ryd})$, as the mixed-source spectrum above.

in expansion, or if they retain thermal memory of earlier epochs when Compton cooling off the microwave background was important, the ratios Si IV/C IV vs C II/C IV may be increased by as much as a factor of 2 at low values of $\mathrm{C}$ II/C IV (high values of $U$ ). It, as suggested by Haehnelt et al. (1997). the temperatures of the clouds are larger than that expected from photoionization thermal equilibrium, the $\mathrm{Si} I V$ fraction is more temperature-sensitive than the $C$ IV fraction, and Si IV/C IV is decreased further relative to $\mathrm{C} \mathrm{IV/}$ $C$ IV. These higher temperatures, suggested as a way to increase the apparent thickness of the clouds, also imply much smaller metallicities, as the C IV fraction is decreased much less compared to the $\mathrm{H}$ I fraction by higher temperatures. For example, adopting the $J_{0, f}$ spectrum and alssuming $U$ $=10^{-2}$, we find that increasing the cloud temperature from 25000 to $70000 \mathrm{~K}$ decreases the C IV fraction by less than $20 \%$ but reduces the $\mathrm{H}$ I fraction (and inferred metallicity) by a factor of 8 .

There is a further effect if the assumed temperature of the cloud is increased to the point that collisional ionization of $\mathrm{H}$ I becomes important. In this case, $\mathrm{He} 1 \mathrm{l} / \mathrm{H} I$ may be larger than that assuming pure photoionization only, $N(\mathrm{He}$ II) may exceed $1 / \sigma_{H c}$, and the cloud maly become self-shielding. Even if this is not the case for a cloud with $N(\mathrm{H} 1)$ $=10^{15} \mathrm{~cm}^{-2}$, this becomes increasingly likely for greater values of $N(\mathrm{HI})$, so that radiative transfer within the individual clouds becomes important. In Fig. 7 we show the results of models that explore some of these temperature effects but defer a complete discussion to a later paper.

\section{RESULTS}

\section{I The Metagaleatic Rudiation Background}

Figure 2 summarizes the results of our photoionization models which assume different sources of photoionizing ra-

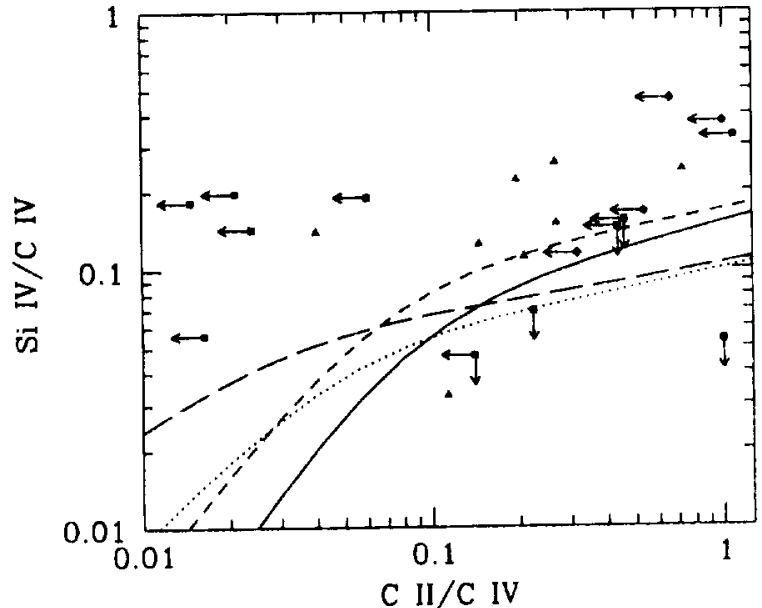

FIG. 2. Ratios $\mathrm{Si}$ IV/C IV vs $\mathrm{C}$ IV/C IV in photoionized models with $N(\mathrm{H} \|$ $=10^{15} \mathrm{~cm}^{2}$. Curves match the spectra of Fig. 1. Triangles are from measured column densities of Si IV, C IV, and C Il (Songaila \& Cowie 1996a. 1996b). Syuares are measurements and upper limits from Songaila \& Cowie (1996a. 1996b). Diamonds are measurements and upper limits from Savaglio et al. (1997). Same data are also used in Figs. 4, 6, 7, 8 with model calculations of $\mathrm{Si}$ iv/C IV vs $\mathrm{C} \mathrm{u} / \mathrm{C} \mathrm{v}$.

diation for the metagalactic background. The solid and dotted curves both assume photoionization by sources that possess the same power-law spectral shape $\left(\alpha_{s}=1.8\right)$ but differ in the treatment of the filtering of their radiation by intervening clouds (see Fig, 1). Models incorporating a pure-AGN spectrum, $J_{Q, f}$, indicate that the Si JV/C IV ratio may be enhanced by almost a factor of 2 over the simpler model for the absorbers with $\mathrm{C}$ II/ $\mathrm{C}$ IV $\geq 0.1$. Even in this regime, $\mathrm{Si}$ is still overabundant relative to $\mathrm{C}$, as previously argued by Songaila \& Cowie (1996a). However, a factor of two enhancement may be sufficient. If the absorbers are photoionized, an increasing $\mathrm{C} \mathrm{II} / \mathrm{C}$ IV ratio is directly related to a decreasing ionization parameter (Fig. 2). At high $\mathrm{C} I 1 / \mathrm{C}$ IV (low $U$ ) there are few high-energy photons available to ionize the higher ionization stages of silicon. As a result, the enhanced ionization of Si III by He II Ly $\alpha$ photons emitted by clouds is enough to increase the ratio of Si IV/C IV over that of the broken power-law models. Compared to more realistic calculations with cloud reemission, models for the spectral shape of the metagalactic background with a simple break at 4 Ryd underestimate the number of high-energy photons in the metagalactic background. At low $\mathrm{C}$ II/C IV (high $U$ ), the Si iv fraction is strongly depleted by the increased amount of high-energy photons in the realistic spectrum.

A metagalactic ionizing background dominated by stellar sources, $J_{\mathrm{MB}}=J_{S Q, f}$, increases the $\mathrm{Si} I V / \mathrm{C} I V$ ratios at $\mathrm{C} \mathrm{II} / \mathrm{C} \mathrm{IV}>0.1$ by $20 \%-50 \%$ over that of the $J_{\mathrm{MB}}=J_{Q, f}$ case. Once again, using a more realistic spectrum increases the Si IV/C IV ratios by almost a factor of 2 compared to spectra with simple attenuation of He II ionizing photons. As in the pure AGN case, the simple representation overestimates the corresponding intensity at higher energies. To be compatible with observed Si IV/C IV ratios if $\mathrm{C} I / \mathrm{C}$ IV $>0.1$, the relative abundance of $\mathrm{Si}$ must be increased by approximately a factor of 2 . In all cases. the large Si IV/C IV ratios in absorbers with 


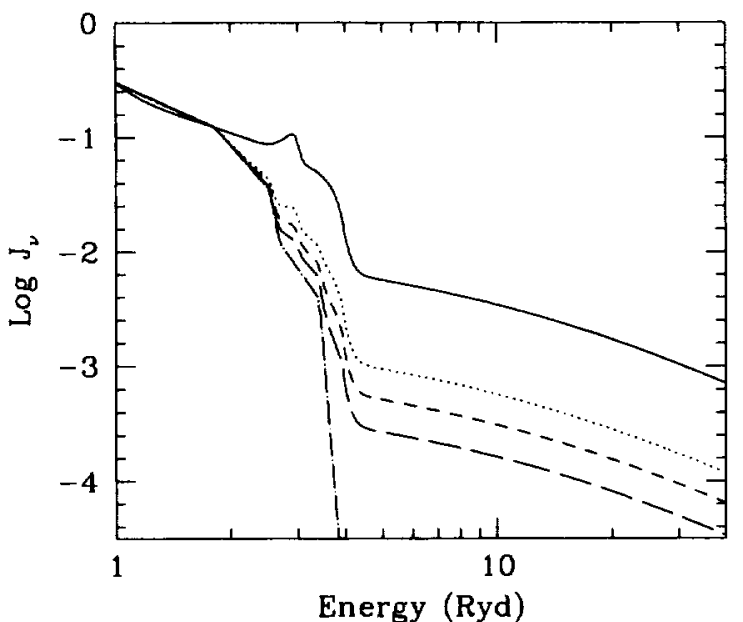

FIG. 3. Mean intensity, in relative units, vs energy in Ryd. All curves have been normalized to the same values at $\nu=\nu_{\mathrm{H}}$. All curves are mixtures where $J_{\mathrm{MB}}$ is the IGM-filtered AGN spectrum shown Fig. 1 , and $J_{L}$ is the unfiltered ("local") stellar spectrum (Sutherland \& Shull 1997). Curves assume $J_{L} / J_{\mathrm{MB}}=0$ (solid), 5 (dotted), 10 (short-dashed), and 20 (longdashed). Dot-long-dashed curve assumes only local stellar radiation, $J_{L}$.

$\mathrm{C} \mathrm{II} / \mathrm{C}$ IV $<0.1$ are difficult to explain in the context of singlephase models unless $\mathrm{Si} / \mathrm{C}$ is enhanced by an order of magnitude. Chemical evolution models with $\mathrm{Si} / \mathrm{C} \geqslant 3(\mathrm{Si} / \mathrm{C})_{\odot}$ are unrealistic, even if the nucleosynthesis is dominated by massive stars (Woosley \& Weaver 1995).

\subsection{A Photoionizing Background Dominated by Local Sources}

One resolution to the puzzle of large Si IV/C IV at small $\mathrm{C}$ II/C IV may be that the ionizing radiation incident on the absorbers is dominated by stellar sources of radiation. As Fig. 4 shows, if the ionizing spectrum is entirely stellar, very large ratios of $\mathrm{Si}$ IV/C IV are always possible due to the steep dropoff in radiation more energetic than $45 \mathrm{eV}$. In practice, however, as Figs. 3 and 4 show, if the metagalactic back-

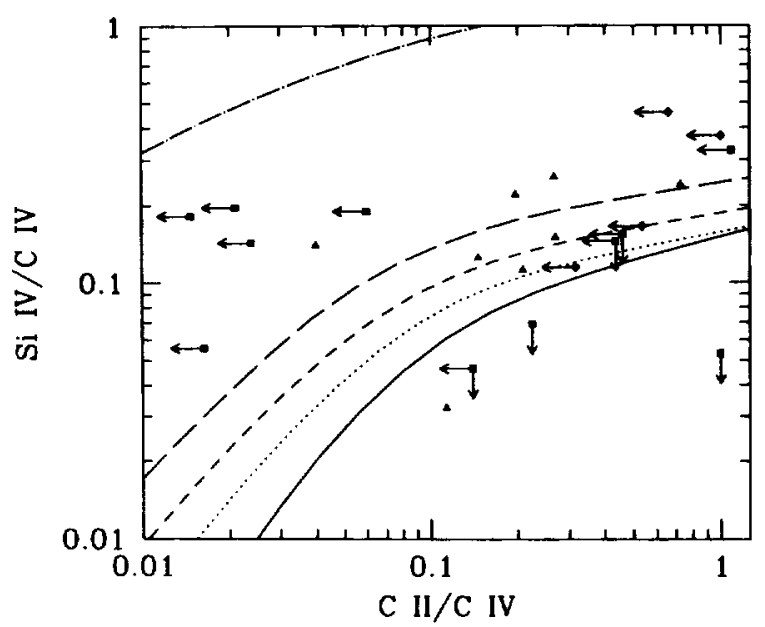

FIG. 4. Ratios of Si IV/C IV vs $\mathrm{C}$ II/C IV in photoionized models with $N(\mathrm{H})=10^{15} \mathrm{~cm}^{2}$. Curves match the spectra of Fig. 3.

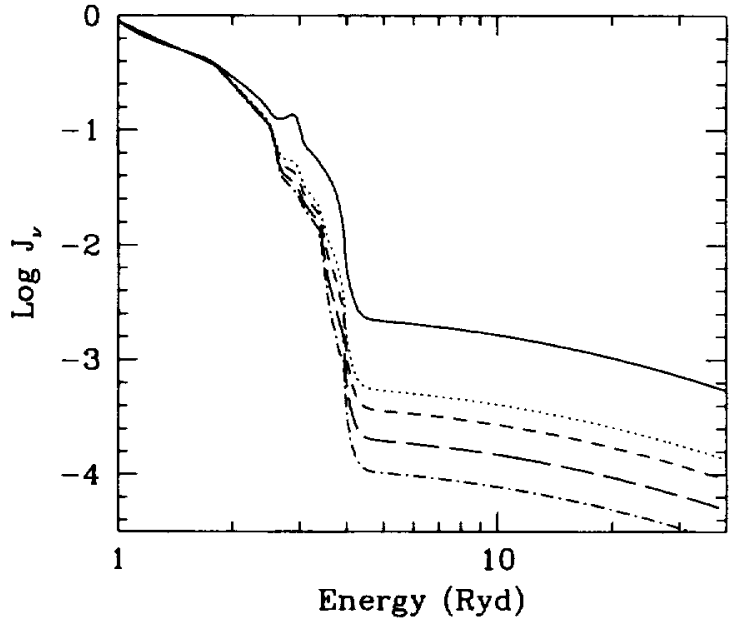

FIG. 5. Mean intensity, in relative units, vs energy in Ryd, where all curves have been normalized to the same values at $\nu=\nu_{\mathrm{H}}$. All curves are mixtures where $J_{\mathrm{MB}}$ is the filtered stellar/AGN spectrum shown in Fig. 1 , and $J_{L}$ is the unfiltered stellar spectrum from Sutherland \& Shull (1997). Curves assume $J_{L} / J_{\mathrm{MB}}=0$ (solid), 3 (dotted), 5 (short-dashed), 10 (long-dashed), and 20 (dot-dashed)

ground is primarily due to quasars $\left(J_{\mathrm{MB}}=J_{Q, f}\right)$, even if $J_{L} / J_{\mathrm{MB}}=20$, high $\mathrm{Si} I \mathrm{~V} / \mathrm{C}$ IV ratios at low $\mathrm{C}$ II $/ \mathrm{C}$ IV require $\mathrm{Si}$ enhancements of a factor of 10 .

If the metagalactic background is dominated by stellar sources $\left(J_{\mathrm{MB}}=J_{S Q, f}\right)$, large enhancements of Si may be unnecessary (see Figs. 5 and 6). For many absorbers with $\mathrm{C} \mathrm{II} / \mathrm{C} \mathrm{IV}>0.1$, no overabundance of $\mathrm{Si}$ is necessary if $J_{L} / J_{\mathrm{MB}}=3-10$. At low $\mathrm{C}$ Il/C IV, $J_{L} / J_{\mathrm{MB}}$ must exceed 10 if enhancements in Si exceeding 2 are to be avoided. As our order of magnitude estimates in Sec. 2.1 indicate, this would require that the absorbers lie within $30 \mathrm{kpc}$ of starburst galaxies. However, this situation is quite feasible, given realistic constraints on absorber size and metal transport distances.

\subsection{Nonequilibrium Temperatures and Nonuniform Radiation Fields}

As Figs. 7(a) and 7(b) show, if the temperatures of the clouds are cooler than the photoionization thermal equilib-

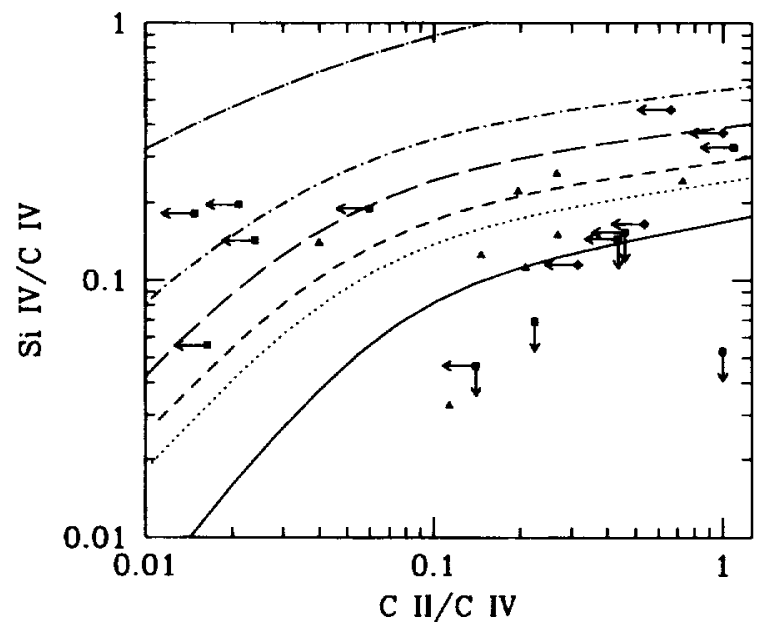

FlG. 6. Ratios Si IV/C IV vs $\mathrm{C}$ II/C IV in photoionized models with $N(\mathrm{H} \mathrm{I})$ $=10^{15} \mathrm{~cm}^{-2}$. Curves match the spectra of Fig. 5. Dot-long-dashed curve assumes only local stellar radiation, $J_{L}$. 


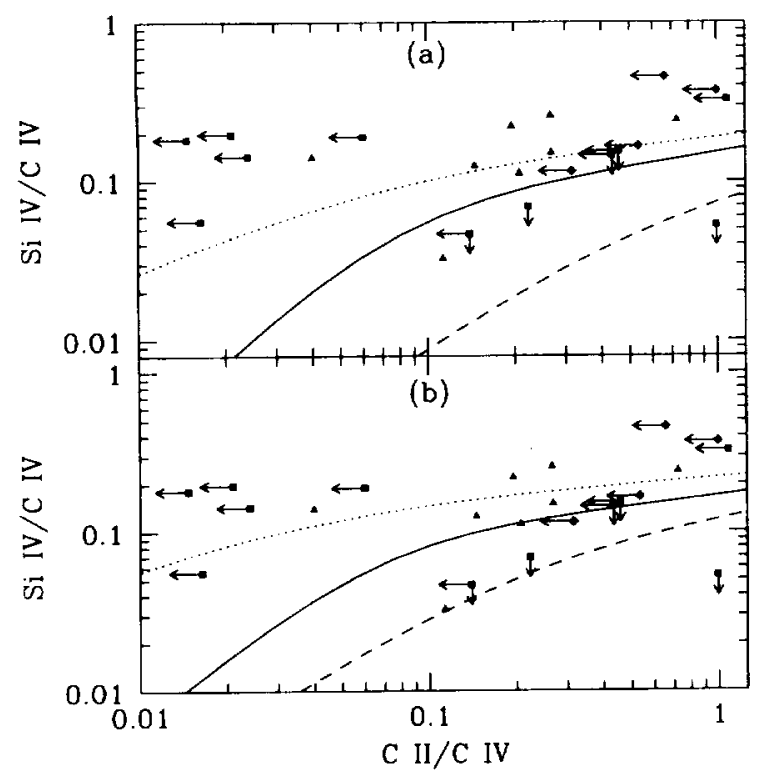

FIG. 7. (a) Effects of varying cloud temperature. All cloud models assume a metagalactic hackground with quasar sources only, $J_{\mathrm{MB}}=J_{Q, t}$. Solid curve adopts the equilibrium temperature computed by CLOUDY for a given ionization parameter and spectral shape (varies between $T=16.500 \mathrm{~K}$ and $T=45000 \mathrm{~K}$. ) Dotted curve assumes constant $T=15(100) \mathrm{K}$ for all models: dashed curve assumes constan $T=50000 \mathrm{~K}$ for all models. (b) Same as (a). except hackground includes both quasars and hot stars, $J_{\mathrm{MB}}=J_{S Q \text {, }}$

rium temperatures (Ferrara \& Giallongo 1997; Zhang et al. 1997), much higher ratios of Si IV/C IV are possible for low $\mathrm{C}$ II/C IV (high $U$ ) clouds. These high $U$ clouds are precisely the clouds for which the photoionization thermal equilibrium temperature is highest ( $T \approx 40000 \mathrm{~K}$ ). The models denoted by the dotted curves in Figs. $7(\mathrm{a})$ and $7(\mathrm{~b})$ assume $T$ $=15000 \mathrm{~K}$, which may not be compatible with the observed linewidths, and which are cooler than is usually assumed for Ly $\alpha$ forest clouds. If higher temperatures are associated with the clouds, for example the dashed curves in Figs. 7(a) and 7(b), which assume $T=50000 \mathrm{~K}$, it is more difficult to account for the ratios in a one-phase model.

Another way to enhance $\mathrm{Si} I V / \mathrm{C} \mathrm{IV}$ at low $\mathrm{C} \mathrm{II/C} \mathrm{IV,} \mathrm{as}$ suggested by Songaila \& Cowie (1996a) and Savaglio et al. (1997), is to assume that no photons above 4 Ryd are present in the incident ionizing spectrum. This is the limiting case of a cloud embedded in a region where He II I-fronts have not yet overlapped. The Si IV/C IV ratios in Fig. 8 are not as high as those for the limiting case when all incident radiation is starburst radiation, since radiation between 45 and $54 \mathrm{eV}$ is included, which will ionize Si IV to Si V. This effect is seen in comparing models which assume pure AGN and starburst/ AGN sources in Fig. 8. As Fig. 8 also shows, a careful treatment of the propagation of these He II I-fronts is necessary, particularly because most AGN possess $x$-ray emission with spectra that flatten to approximately $\nu^{-1}$ above $0.3 \mathrm{keV}$. We have shown that including high-energy radiation with $E$ $>25 \mathrm{Ryd}$ is sufficient to reduce $\mathrm{Si}$ IV/C IV ratios substantially at low $\mathrm{C} \mathrm{II/C} \mathrm{IV.}$

\section{DISCUSSION}

From the results of our one-phase photoionization models we draw the following conclusions.

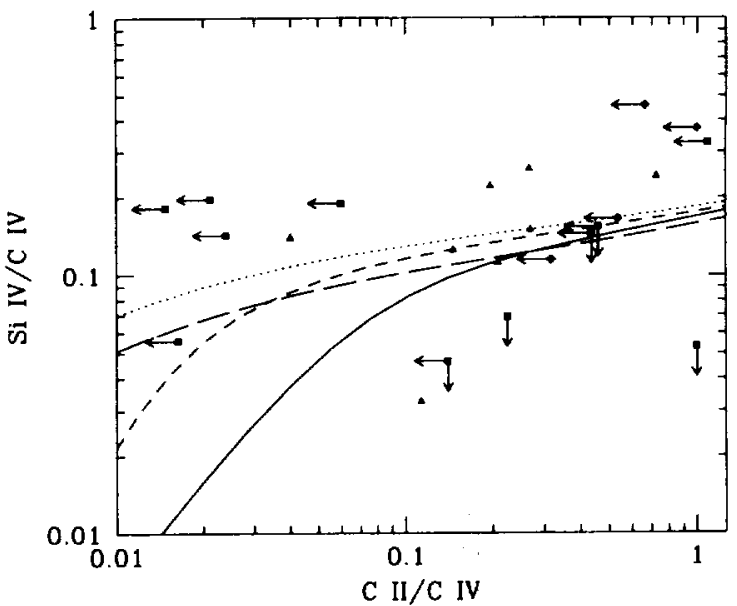

FIG. 8. Ratios Si IV/C IV vs $\mathrm{C} I / \mathrm{C}$ IV in an IGM in which $\mathrm{He}$ II ionization fronts have not overlapped. Solid curve repeats models for the filtered starburst/AGN spectrum in Fig. 2. Dotted assumes filtered starburst/AGN spectrum with all photons with $E>4$ Ryd removed. Long-dashed curve assumes $v^{-1.8}$ AGN spectrum with all photons with $E>4$ Ryd removed. Short-dashed curve assumes filtered starburst/AGN spectrum with $4<E<25$ Ryd photons removed.

(1) Ionizing Radiation Field. - The metagalactic radiation field is likely to include both power-law (AGN) and stellar (hot-star) components. The Ly $\alpha$ forest absorbers with metal lines may also experience a local radiation field from starburst galaxies within $50-100 \mathrm{kpc}$.

(2) $\mathrm{Si} / \mathrm{C}$ Overabundance.-For plausible mixtures of stellar and AGN spectra in the metagalactic background, our photoionization models produce enhanced $\mathrm{Si}$ IV/C IV ratios, consistent with high-z absorbers with $\mathrm{Si} / \mathrm{C} \approx 2(\mathrm{Si} / \mathrm{O})_{0}$ at low ionization ( $\mathrm{C} \mathrm{II} / \mathrm{C}$ IV $>0.1$ ). For absorbers with $\mathrm{C} \mathrm{II} / \mathrm{C}$ IV $<0.05$, it is difficult to account for the high values of Si IV/ $\mathrm{C}$ IV unless $\mathrm{Si} / \mathrm{C}>10(\mathrm{Si} / \mathrm{C})$, an unrealistically large value for massive-star nucleosynthesis. These systems may include photoionization from local stellar sources as well as hot, collisionally ionized gas.

(3) Local lonizing Sources. - If the radiation field incident on the absorbers is dominated by a nearby starburst galaxy, the $\mathrm{Si}$ IV/C IV ratios are further enhanced. If $\mathrm{C}$ II/C IV $>0.1$, no $\mathrm{Si} / \mathrm{C}$ overabundance is necessary to explain $\mathrm{Si}$ IV/C IV ratios if absorbers are within about $40 \mathrm{kpc}$ of a starburst galaxy and the background is dominated by stellar sources. Even if the metagalactic background is dominated by AGNs, close proximity to a starburst galaxy may reduce the needed $\mathrm{Si} / \mathrm{C}$ overabundance to a factor 1.5 . When $\mathrm{C} 1 \mathrm{I} / \mathrm{C}$ IV $\leqslant 0.05$, it remains very difficult to account for the high values of Si IV/ C IV with photoionized models, although models with locally dominated radiation may only require $\mathrm{Si} / \mathrm{C}$ enhancements of a factor of 2-3.

(4) Non-Overlapping He II l-Fronts-Around quasars whose ionizing continua have steep spectral indices ( $\alpha_{s}$ $>1.84$ ), the He II I-fronts will lie within the H I I-fronts. If many absorbers lie in regions where all photons above 4 Ryd are attenuated (Songaila \& Cowie 1996a), we obtain good agreement with almost all measurements of $\mathrm{Si}$ IV/C IV if $\mathrm{Si} / \mathrm{C}$ is enhanced by a factor of 2-3. However, including even a small contribution of higher energy photons in the back- 
ground increases the needed $\mathrm{Si} / \mathrm{C}$ overabundance to an order of magnitude for absorbers with the lowest $\mathrm{C}$ u/C IV ratios.

(5) Temperature Effects.-If the absorbers are cooler than expected for thermal equilibrium between photoelectric heating and radiative cooling, the $\mathrm{Si} I V / C$ IV ratio is increased for a given $\mathrm{C} \mathrm{II/C}$ IV ratio. At low $\mathrm{C} \mathrm{II} / \mathrm{C}$ IV, this enhances Si IV/ $C$ IV by a factor of 5 in our photoionization models. Conversely, if the absorbers are hotter, Si IV/C IV is lower for a given $\mathrm{C}$ II/C IV ratio.

While we have described several processes that might increase the Si IV/C IV ratio, an important additional constraint on these possibilities is the fact that the high ratios are preferentially found at $z>3.1$. Recent observations (Boksenberg 1997) challenge this interpretation, by finding high Si IV/C IV ratios in absorbers at $z=2-3$. If, however, Si IV/C IV rises above $z>3.1$, this argues for a time dependence to whatever process increases this ratio. It is this property, as well as the increased He II absorption toward Q0302-003 at $z=3.28$ (Jakobsen et al. 1994), that Songaila \& Cowie (1996a) use to support their suggestion that the absorbers lie in regions where He II l-fronts have not overlapped at $z>3.1$. From a higher resolution $H S T / G H R S$ spectrum of Q0302-003, Hogan et al. (1997) find evidence for residual transmitted flux below the He II edge. Their $95 \%$ confidence upper limit, $\tau_{\text {He } 11} \leqslant 3$, makes it less necessary to propose that Q0302-003 lies in a region where $\mathrm{He}$ II I-fronts have not overlapped, but does not preclude the suggestion that such regions existed at $z>3.1$. If this is the case, radiation outside of He Ill regions would largely limit the range in ionization stages for carbon to II-IV, and for silicon to II-V. In AGN, however, the large fluxes of unattenuated photons above $45 \mathrm{eV}$ can ionize Si IV and $\mathrm{C}$ III, so that high observed Si IV/C IV ratios would be difficult to explain for large $U$ (small $\mathrm{C}$ IV/C IV). As a result, the spectrum below 4 Ryd must be dominated by stellar sources. This may be difficult to achieve with the known quasar luminosity functions at $z>3.5$. In addition, soft $x$-ray radiation from quasars, which is less attenuated by intervening clouds even in the low He III porosity case, may again make high Si IV/C IV, low C IV/C IV absorbers difficult to understand if they are solely photoionized by a metagalactic background.

New or improved measurements of He II absorption may soon indicate whether He II I-fronts have overlapped well before $z=3.1$. In that case, a sharp change in the shape of the metagalactic radiation field may be less plausible. Still, in general, the trend is likely to be more attenuation of photons above the 4 Ryd limit with increasing redshift. Other time dependent effects that enhance the ratio of Si IV/C IV are possible. Higher abundances of $\mathrm{Si}$ relative to $\mathrm{C}$ are associated with metal yields from the most massive stars. However, in a flat universe with $h=0.75$, the age of the universe exceeds $10^{9}$ years by $z=3.1$, time enough for lower-mass stars to enrich the gas with carbon. For example, if multiple supernovae eject metal-enriched gas into the IGM, the additional $4 \times 10^{8}$ years between $z=3.5$ and $z=2.5$ may make it more likely that the gas surrounding the high-mass stars has been enriched with carbon from a previous episode of star formation.

The temperature effects we discuss in Sec. 4 may also have a time dependence. If the IGM has been uniformly enriched by a much earlier episode of Population III star formation, and if the Ly $\alpha$ forest clouds with metals have their origin in growing overdensities in the IGM, then the absorbers observed at higher $z$ may have formed at an earlier epoch. If Compton cooling off the cosmic microwave background was a dominant coolant at this epoch (MiraldaEscudé \& Rees 1994), the cloud may have retained memory of this lower temperature. This effect is probably not large, since there is not a significant evolution in the observed linewidths.

Alternatively, one may resolve the puzzle of the high Si IV/C IV, low $\mathrm{C}$ II/C IV absorbers by relaxing the singlephase model which we used to explore the effects of radiative transfer. As we have emphasized in a previous paper (Giroux et al. 1994), the production of heavy elements in QSO absorption systems is naturally accompanied by hot gas due to supernovae and hot-star winds.

We thank Mark Fardal for the calculated metagalactic background radiation spectra. We also thank G. Ferland for discussions about updated versions of CLOUDY, and $\mathbf{L}$. Cowie and A. Songaila for providing a summary of measurements and upper limits on metal column densities in advance of publication. This work was supported by the Astrophysical Theory Program at the University of Colorado (NASA Grant No. NAGW-766).

\section{REFERENCES}

Bennett, C. L.. et al. 1994, ApJ, 434, 587

Bergeron, J.. \& Stisinska. G. 1986, A\&A. 169, 1

Bergeron, J., \& lkeuchi, S. 1990, A\&A, 235, 8

Boksenberg, A. 1997 (private communication)

Bruzual, G. \& Charlot. S. 1993. ApJ. 405, 538

Cooke, A. J. Espey, B., \& Carswell. B. 1997, preprint, astro-ph/96091(10)

Copi. C. J.. Schramm, D. N., \& Turner, M. S. 1994, Science, 267. 192

Couchman. H. M. P. 1985. MNRAS. 214. 137

Cowie. L. L., Songaila. A., Kim, T.-S., \& Hu, E. M. 1995, AJ. 109,1522

Donahue, M.. \& Shull, J. M. 1987, ApJ, 323, 1.13

Donahue. M., \& Shull. J. M. 1991, ApJ, 383, 511

Dove. J. B.. \& Shull. J. M. 1994. ApJ, 430, 222

Fardal. M. A. Giroux, M. L.. \& Shull, J. M. 1997, ApJ (submitted)

Ferland, G. J. 1996, Haxy, a Brief Introduction to Cloudy, University of

Kentucky Department of Physics and Astronomy Internal Report
Ferrara, A., \& Giallongo, E. 1997, preprint

Gabler, R., Gabler, A., Kudritzki, R. P., \& Mendez, R. H. 1992. A\&A. 265. 6.56

Giroux, M. L., Fardal, M. A., \& Shull, J. M. 1995, ApJ. 451, 477

Giroux, M. L., \& Shapiro, P. R. 1996, ApJS, 102, 191

Giroux, M. L., Sutherland, R. S. \& Shull, J. M. 1994, ApJ. 435. L97

Haardt, F., \& Madau, P. 1996, ApJ, 461, 20

Haehnelt, M. G. 1997, preprint, astro-ph/9608009

Haehnelt, M. G., Rauch, M., \& Steinmetz, M., 1997, preprint, astro-ph/ 9608133

Hellsten, U., Davé, R.. Hernquist. L., Weinberg, D. H., \& Katz. N. 1997. preprint, astro-ph/970!043

Hogan. C. J., Anderson, S. F., \& Rugers, M. H. 1997. AJ, 113, 1495

Jakobsen. P.. Boksenherg, A., Deharveng, J. M., Greenfield, P. Jedrzejew'ski, R. \& Paresce, F. 1994, Nalure, 370, 35 
Kurucz, R. L. 1992, in The Stellar Populations in Galaxies, IAU Symposium 149, edited by B. Barbuy and A. Renzini (Kluwer, Dordrecht), p. 225

Madau, P., \& Meiksin, A. 1994, ApJ, 433, L53

Madau, P., \& Shull. J. M. 1996, ApJ, 457, 551

Madau, P. 1992, ApJ, 389, LI

Miralda-Escudé, J., \& Ostriker, 1990, ApJ, 350, I

Miralda-Escudé, J., \& Rees, M. J. 1994, MNRAS, 266, 343

Olive, K. A., \& Steigman, G. 1995, ApJS, 97, 49

Ostriker, J. P., \& Gnedin, N. Y. 1996, ApJ, 472, L63

Paresce, F., Mckee, C. F., \& Bowyer, S. 1980, ApJ, 240, 387

Petitjean, P., Riediger, R., \& Rauch, M. 1996, A\&A, 307, 417

Sargent, W. L. W., Boksenberg, A., \& Steidel, C. C. 1988, ApJS, 68, 539

Savaglio, S., Cristiani, S., D'Odorico, S., Fontana, A., Giallongo, E., \& Molaro, P. 1997, astro-ph/9606063

Schaerer, D., \& de Koter, A. 1996, preprint, astro-ph/9611068
Sellmaier, F. H., Yamamoto, T., Pauldrach, A. W. A., \& Rubin, R. H. 1996,A\&A, 305, L37

Skillman, E. D., \& Kennicutt, R. C. 1993, ApJ, 411, 655

Shapiro, P. S., \& Giroux, M. 1987, ApJ, 321, L107

Songaila, A., \& Cowie, L. L. 1996a, AJ, 112, 335

Songaila, A., \& Cowie, L. L. 1996b, personal communication

Steidel, C. C., Sargent, W. L. W., \& Boksenberg, A. 1988, ApJ, 333, 5

Steidel, C. C., \& Sargent, W. L. W. 1989, ApJ, 343, L.33

Sutherland, R. S., \& Shull, J. M. 1997, in preparation

Tytler, D., Fan, X.-M., Burles, S., Cottrell, L., Davis, C., Kirkman, D., \& Zuo, L. 1995, in ESO workshop on QSO Absorption Lines, edited by $G$. Meylan (Springer, Heidelberg), p. 289

Woosley, S. E., \& Weaver, T. W. 1995, ApJS, 101, 181

Zhang, Y., Anninos, P., Norman, M. L., \& Meiksin, A. 1997, preprint Zheng, W., Kriss, G. A., Telfer, R. C., Grimes, J. P., \& Davidsen, A. F. 1997, ApJ (in press) 
